\title{
Deuteration Effect on the Coexistence Curves of Semidilute Polymer Solutions
}

\author{
Takashi ICHIMURA, Koji OKANO,* Kimio KuRITA, \\ and Eiichi WADA \\ College of Science and Technology, Nihon University, \\ Kanda-Surugadai, Chiyoda-ku, Tokyo 101, Japan \\ * Department of Applied Physics, Faculty of Engineering, \\ University of Tokyo, Hongo, Bunkyo-ku, Tokyo 113, Japan
}

(Received March 30, 1987)

\begin{abstract}
Phase separation points of semidilute solutions of polystyrene in deuterated cyclohexane were measured and compared with those of polystyrene in protonated cyclohexane. Theoretical calculation of the coexistence curve by using the interaction parameters extracted from small-angle scattering of neutron and X-rays has revealed that the deuteration effect on the coexistence curve is due solely to a shift of $\theta$ point, the temperature at which the binary cluster integral vanishes, while leaving other factors unaltered. The origin of this feature is briefly discussed.
\end{abstract}

KEY WORDS Coexistence Curve / Deuteration Effect / Semidilute Solutions / Polystyrene / Deuterated Cyclohexane / Poor Solvent Regime /

Small-Angle Neutron Scattering /

In the preceding paper ${ }^{1}$ we discussed the coexistence curve of polystyrene in cyclohexane in the semidilute range. We compared the measured phase separation points with theoretical coexistence curve using the interaction parameters deduced from small-angle $\mathrm{X}$ ray scattering (SAXS) and found good agreement between them.

On the basis of this, we discuss in this paper the deuteration effect on the coexistence curve: we measure the phase separation points of polystyrene (PS) in deuterated cyclohexane $\left(\mathrm{C}_{6} \mathrm{D}_{12}\right)$ and compare them with those of PS in protonated cyclohexane $\left(\mathrm{C}_{6} \mathrm{H}_{12}\right)$.

In section of MEASUREMENT OF PHASE SEPARATION POINTS we describe the procedure and results of the measurement. In section of THEORETICAL COEXISTENCE CURVE, we calculate the theoretical coexistence curve of PS- $\mathrm{C}_{6} \mathrm{D}_{12}$ system in the semidilute range and compare it with measured phase separation points. In section of DISCUSSION, we discuss briefly the origin of the differences of the coexistence curves of the deuterated and protonated solvents.

\section{MEASUREMENT OF PHASE SEPARATION POINTS}

The PS samples were obtained from Pressure Chemical Co. and Toyo Soda Inc. The samples are characterized by $\bar{M}_{w} /$ $\bar{M}_{n} \leqq 1.06$ for $M=3.0 \times 10^{5}$ and $\bar{M}_{w} / \bar{M}_{n} \leqq 1.05$ for $M=1.26 \times 10^{6}$ and $\bar{M}_{w} / \bar{M}_{n} \leqq 1.04$ for $M=$ $3.84 \times 10^{6}$. These PS samples were dried for three days in a vacuum box. $\mathrm{C}_{6} \mathrm{D}_{12}(99.5$ atom $\%$ deuterated) was used as the solvent. Flame sealed Pyrex tubing cells (inner diameter $8 \mathrm{~mm}$ ) containing the sample solution were immersed in a water thermostat controlled to $\pm 0.03^{\circ} \mathrm{C}$. 


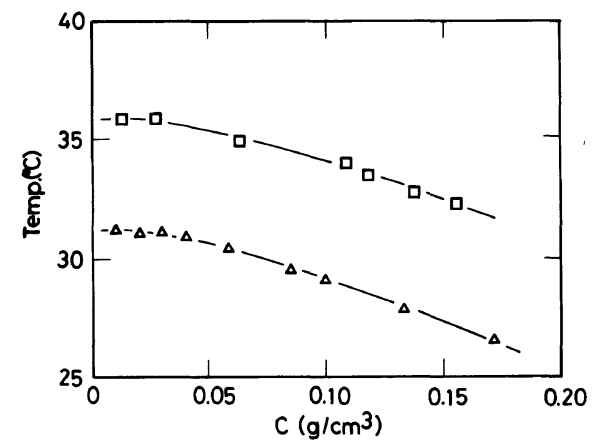

Figure 1. Measured phase separation points for $M=$ $3.84 \times 10^{6} .(\square) \mathrm{PS}-\mathrm{C}_{6} \mathrm{D}_{12} ;(\triangle) \mathrm{PS}-\mathrm{C}_{6} \mathrm{H}_{12} \cdot{ }^{1}{ }^{1}$

Phase separation points were determined optically as reported in the preceding paper ${ }^{1}$ with an accuracy of $\pm 0.05^{\circ} \mathrm{C}$ by observing the $\mathrm{He}-\mathrm{Ne}$ laser beam transmitted through the solution cell.

Typical measured phase separation points of PS in $\mathrm{C}_{6} \mathrm{D}_{12}$ system are shown in Figure 1 together with those of PS in $\mathrm{C}_{6} \mathrm{H}_{12}$ system reported in the preceding paper. ${ }^{1}$ In spite of the same molecular weight of PS the phase separation temperatures of the deuterated system are a little higher than those of the protonated system.

\section{THEORETICAL COEXISTENCE CURVE}

As described already ${ }^{2}$ the semidilute branch of the theoretical coexistence curve can be obtained by equating the osmotic pressure $\pi$ to zero. ${ }^{3}$ In a mean field approximation we have

$$
\frac{\pi}{N_{\mathrm{A}} k_{\mathrm{B}} T}=\frac{c}{M}+\frac{1}{2} B_{1} N_{\mathrm{A}} c^{2}+B_{2} N_{\mathrm{A}}^{2} c^{3}=0
$$

where $c$ is the weight concentration $\left(\mathrm{g} \mathrm{cm}^{-3}\right)$, $M$ the molecular weight of the polymer, $B_{1}$ and $B_{2}$ are the binary and ternary cluster integrals of the polymer segment respectively, $N_{\mathrm{A}}$ is Avogadro's number and $k_{\mathrm{B}} T$ has the usual meaning.

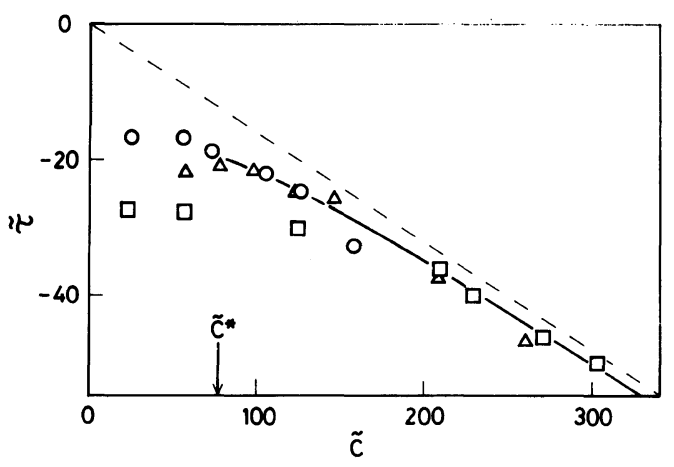

Figure 2. Theoretical coexistence curve (solid line) and observed phase separation points. (O) $M=3.0 \times 10^{5}$; ( $\triangle$ ) $M=1.26 \times 10^{6}$; ( $\left.\square\right) M=3.84 \times 10^{6}$. Broken line represents the asymptote to the theoretical coexistence curve.

On the other hand, by means of small-angle neutron scattering (SANS) we measured the interaction parameters for PS- $\mathrm{C}_{6} \mathrm{D}_{12}$ system near the $\theta$ point. $^{4, \dagger}$ The results are summarized as

$$
\begin{aligned}
& B_{1}=B_{10} \tau, \quad B_{10}=6 \times 10^{-27} \mathrm{~cm}^{3} \\
& \tau \equiv 1-\theta / T, \quad \theta=313 \mathrm{~K} \\
& B_{2}=0.8 \times 10^{-51} \mathrm{~cm}^{-6}
\end{aligned}
$$

This set of interaction parameters gives the theoretical coexistence curve ${ }^{2}$

$$
\tilde{\tau}=-5.6 \times 10^{2}\left(\frac{1}{\tilde{c}}\right)-0.16 \tilde{c}
$$

where

$$
\begin{aligned}
& \tilde{\tau} \equiv \sqrt{M} \tau \\
& \tilde{c} \equiv \sqrt{M} c
\end{aligned}
$$

As shown in Figure 2, this theoretical curve reproduces the observed phase separation points quite accurately. In this figure, experimental points for two other samples having different molecular weights $\left(M=3.0 \times 10^{5}\right.$ and $M=1.26 \times 10^{6}$ ) are also included. As for the PS- $\mathrm{C}_{6} \mathrm{H}_{12}$ system, SAXS measurement re-

$+\theta$ point in this paper and also in ref 1,2 , and 4 is defined as the temperature at which the binary cluster integral vanishes. 
vealed that the interaction parameters are given respectively by the same equations as eq 2,3 , and 4 except for the $\theta$ temperature. $^{1}$ The $\theta$ temperature was found to be $308 \mathrm{~K}^{1}$ Therefore eq 5 describes equally well the semidilute branch of the coexistence curve of the PS- $\mathrm{C}_{6} \mathrm{H}_{12}$ system.

We thus realize that the deuteration effect on the coexistence curve is due solely to the shift of $\theta$ temperature without changing the values of $B_{10}$ and $B_{2}$.

\section{DISCUSSION}

In order to understand qualitatively the effect of the deuterium substitution of the solvent on the interaction parameters, let us discuss $B_{1}$ and $B_{2}$ as given by the lattice model $^{3}$ :

$$
\begin{gathered}
B_{1}=m^{-1 / 2} A^{3}(1-2 \chi) \\
B_{2}=m^{3} A^{6}
\end{gathered}
$$

where $m$ is the molecular weight of polymer segment and $A M^{1 / 2}$ is the unperturbed end-toend distance. Considering the contribution of the dispersion forces, the parameter $\chi$ is, in the first approximation, proportional to the square of the difference of the polarizability of a polymer segment $\alpha_{p}$ and that of solvent $\alpha_{s}{ }^{3}$ :

$$
\chi=\frac{\kappa}{2 T}\left(\alpha_{\mathrm{p}}-\alpha_{\mathrm{s}}\right)^{2}
$$

where $\kappa$ is a positive constant. Thus, the $\theta$ temperature in this model is given by

$$
\theta=\kappa\left(\alpha_{\mathrm{p}}-\alpha_{\mathrm{s}}\right)^{2}
$$

whereas $B_{10}$ is given by

$$
B_{10}=m^{-1 / 2} A^{3}
$$

Therefore, the above mentioned deuteration effect, i.e., a slight shift of $\theta$ temperature leaving the values of $B_{10}$ and $B_{2}$ unaltered, can be explained as due to small difference in the polarizability of $\mathrm{C}_{6} \mathrm{H}_{12}$ and $\mathrm{C}_{6} \mathrm{D}_{12}$. This slight shift of polarizability might be associated with a small contribution of nuclear motions of hydrogen atoms to the polarizability of the solvent in the infrared region. This conclusion is in agreement with the consideration by Strazielle and Benoit ${ }^{5}$ who explained the deuteration effect on the $\theta$ temperature on the basis of solubility parameters.

\section{REFERENCES}

1. T. Ichimura, K. Okano, K. Kurita, and E. Wada, Polymer, 28, 1573 (1987).

2. K. Okano, T. Ichimura, K. Kurita, and E. Wada, Polymer, 28, 693 (1987).

3. P. G. de Gennes, "Scaling Concepts in Polymer Physics," Cornell University Press, Ithaca, New York, 1979.

4. K. Okano, K. Kurita, S. Nakajima, E. Wada, M. Furusaka, and Y. Ishikawa, Physica, 120B, 413 (1983).

5. C. Strazielle and H. Benoit, Macromolecules, 8, 203 (1975). 